\title{
USING THE CASE METHOD TO FACILITATE LEARNING OF DESIGN FOR MANUFACTURING AND COST
}

\author{
David Effa*, Oscar Nespoli** and Steve Lambert*** \\ Waterloo Cases in Design Engineering, University of Waterloo, Waterloo, Ontario, Canada \\ Mechanical and Mechatronics Engineering, University of Waterloo \\ deffa@uwaterloo.ca*, oscar@uwaterloo.ca**, steve@uwaterloo.ca***
}

\begin{abstract}
Design for Manufacturing and Assembly (DFMA) is an integral methodology for product development that aims to simplify the manufacturing process, increase productivity, and minimize costs while maintaining product quality. DFMA is often difficult since significant manufacturing knowledge is required. The importance of DFMA is underlined by the fact that a large portion of product manufacturing costs (materials, processing, assembly and indirect costs) is determined by early design decisions. Therefore, it is important for engineering students to understand the limiting factors and practices relevant to the application of DFMA. Although DFMA concepts can be taught through conventional lecture methods, true understanding of this multi-faceted and highly integrated strategy requires real-world practice. The case method provides an effective pedagogical approach to help students understand and fully appreciate the complexity of engineering practice, gain experience and develop the skills necessary to deal with this complexity, and make connections between various topics in their undergraduate curriculum. In this paper we describe the effort taken in Mechanical Engineering at the University of Waterloo (UW) to develop and implement case studies to address this gap.
\end{abstract}

Keywords: Design for Manufacturing (DFM); Product cost; Design for Assembly (DFA); Case method; Design case study; Design education

\section{INTRODUCTION}

Product Design for Manufacturing and Assembly (DFMA) is widely understood to be critical for improving productivity and competitiveness in industry. DFMA is an engineering methodology that bases its principles in designing products in such a way that they can be manufactured with the machines available and at minimum cost and time. This methodology is already well developed in the literature [1-2], which provides information and guidelines for individual manufacturing process, but not for production systems that combine several different components in the flow of operations [1]. Therefore, engineering education, particularly mechanical engineering education, should foster DFMA skills development using both theoretical and practical approaches to prepare students for opportunities and challenges when they enter the workforce.

The case method creates a practical classroom environment in which students absorb facts and theories, but also exercise fundamental engineering skills using real world examples. Cases are also an ideal mechanism for teaching specialized topics, such as DFMA, and the importance of cost considerations in engineering design [3].

The Waterloo Cases in Design Engineering (WCDE) group at the University of Waterloo was established to promote and implement the case study teaching method in the Faculty of Engineering; first by generating cases and then by pervasively implementing them throughout the engineering curriculum. To date, WCDE has developed over 133 engineering cases that illustrate real-world engineering design and demonstrate practical applications of engineering science. WCDE cases have been used in various forms to add new dimensions to the learning process. Where and how cases are used depends on the course objectives, the nature of the class and the instructor. WCDE cases have been incorporated into new courses at an average rate of 15 cases per term.

The primary objective of this paper is to present the use of an engineering case study to facilitate learning of DFMA and estimating product cost. A case was developed from a student work term report, which documented an improved design for a digital camera body. Students used this case study along with a rapid prototype sample of the final product in a third year Mechanical Engineering Design Workshop course (ME 380) during the fall 2013 academic term. As part of small group discussions, a number of different designs for the case problem were suggested and discussed in class. The results of a student survey indicate that the case study was an engaging application and that group discussion and interaction helped to enhance understanding of DFMA and the specific design challenge described in the case. 


\section{Design for Manufacturing and Assembly}

Manufacturing can be defined as a sequence of processes that transform raw or partially processed material into a final product that has value for the customer [4]. These manufacturing processes are chosen during the conceptual design of the product, which means it is necessary for the designers to have a solid understanding of them. The design must meet all pre-defined design constraints, including the manufacturability of the part using the company's or suppliers existing machines and processes. Also, production cost must be minimized in order to achieve the highest profit. These issues underscore the need to implement the DFMA methodology early in the design process.

DFMA is a philosophy and mind-set in which manufacturing input is used at the earliest stages of design to develop parts and products that can be produced easily and economically [5]. DFMA spans every aspect of the design process in which the issues involved in manufacturing a designed object are considered explicitly with a view to influencing product design. Examples include examining tooling costs or time required, processing costs or controllability, assembly time or costs, human concerns during manufacturing (e.g., worker safety or quality of work required) and availability of materials or equipment. Design for Manufacturing occurs - or should occur - throughout the design process. Detailed Design for Assembly Guidelines recommends [6]:

- Simplifying the design and reduce the number of parts

- Standardizing and use of common parts

- Designing for ease of fabrication

- Mistake-proof product design and assembly

- Designing for parts orientation and handling

- Minimizing flexible parts and interconnections

- Designing for ease of assembly

- Designing for efficient joining and fastening

- Designing modular products to facilitate assembly

- Designing for automated production

\section{FOSTERING DFMA SKILLS USING THE CASE METHOD}

Many Engineering schools, Mechanical Departments in particular, teach students about DFMA and manufacturing concepts through hands-on activities in the student shop. However, this approach lacks academic content and, with rapidly evolving manufacturing and product technologies, cannot remain current. By focusing on one or two core examples, this approach also fails to address the full spectrum of DFMA topics. Fourth year design projects are commonly used in post-secondary institutions to cultivate DFMA skills. While providing solid opportunities for practical skills development, this method is limited in scope and may not effectively address real-world issues faced by industry. At the University of Waterloo (UW), student competition teams, such as Formula SAE, Baja, Solar Vehicle and EcoCar, offer DFMA learning experience to those students who participate directly. The team environment offers realistic engineering challenges where technical skills including design, manufacturing and performance skills are developed and tested. However, a limited number of students participate in these extra-curricular activities, which limits the impact of this approach for the majority of students. In addition, this type of low-volume design application does not fully allow DFMA to be realized as important.

The case method allows students to see the complexity of everyday practical challenges in context, appreciate this complexity and develop techniques and judgment to deal with it while at the same time raising their level of engagement in the learning process. The case method creates a classroom environment in which students must not only gain theoretical understanding, but also employ leadership and teamwork skills to address real problems.

Using the case method to achieve specified outcomes requires effort in three domains, as shown in Figure 1. These domains are: development, implementation and assessment. Development entails creating cases and defining measurable learning objectives for these cases. Implementation is the selection and utilization of cases that will be used to deliver the specified content, thus facilitating student achievement of the objectives.

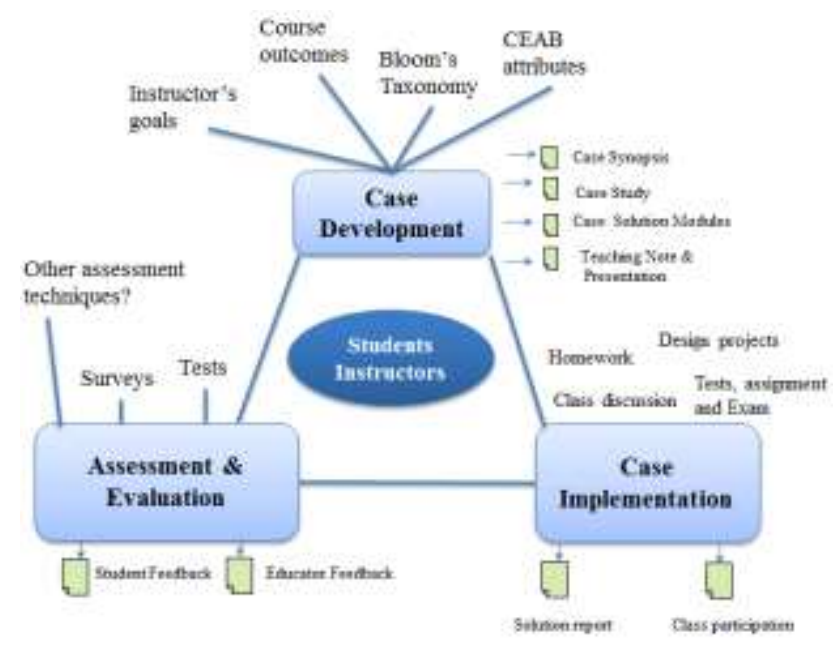

Figure 1: Elements of the case method 
Assessment encompasses selecting and implementing methods that will be used to determine if (and how well) the objectives have been achieved. In order to effectively use the case method, WCDE has built a strong foundation through the development of a comprehensive, diverse and sustainable supply of engineering cases for use throughout the curriculum. WCDE is currently working on more assertive ways to promote implementation. Furthermore, evaluating and assessing the use of the case method in engineering courses is vital for improving case method activities. The evaluation process is directed at maintaining and improving the quality of the program, as well as assessing the benefits of case-based learning for improving student design and non-technical skills.

\section{CASE IMPLEMENTATION}

WCDE cases provide complete details of an engineering problem as well as instructor modules which outline the entire process undertaken by the principal engineer to solve such a problem. Consequently, an engineering case is different from a problem that might be presented in an engineering textbook. Typical WCDE case implementation activities include professor planning, individual preparation by students (assignments), small group (team) discussion, classroom discussion and perhaps a team assignment.

The Dalsa Harmony Camera Body Part Design Case Study was developed in 2013 and intended for use in Mechanical Engineering and System Design courses covering topics such as design, manufacturing and heat transfer. The scope and specific topics covered by the case study are broader than the base material taught in the targeted courses. The case documents the design of a camera cover, the parts fabrication process, and a heat sink thermal analysis by a co-op student working for Teledyne Dalsa.

The student used DFMA methodology to simplify Harmony camera mechanical parts in terms of minimizing the number of parts, and minimizing the time and therefore costs of the assembly process. The case solution modules also cover topics in material selection and cost estimation, presenting trade-off decisions for alternate designs. Case materials include the case study itself, solution modules, teaching note and presentations. Appendix A provides a synopsis of this particular case.

The Harmony design is based on Dalsa's existing camera and specifically targets reduced production costs with comparable performance, scalable component architecture and lower bill of materials (BOM) costs. The project design focuses on the camera assembly, specifically reducing fabrication costs of the Harmony camera body components (H-Section, a 3D printed model of which is shown in Figure 2) while maintaining adequate heat transfer performance. The $\mathrm{H}$-section is the structural housing to which key electronic components are attached, and it transfers heat away from these components via an internal heat sink bridge. In the existing camera, the $\mathrm{H}$-section is manufactured from billet aluminum. A more cost-effective solution was desired for the Harmony camera.

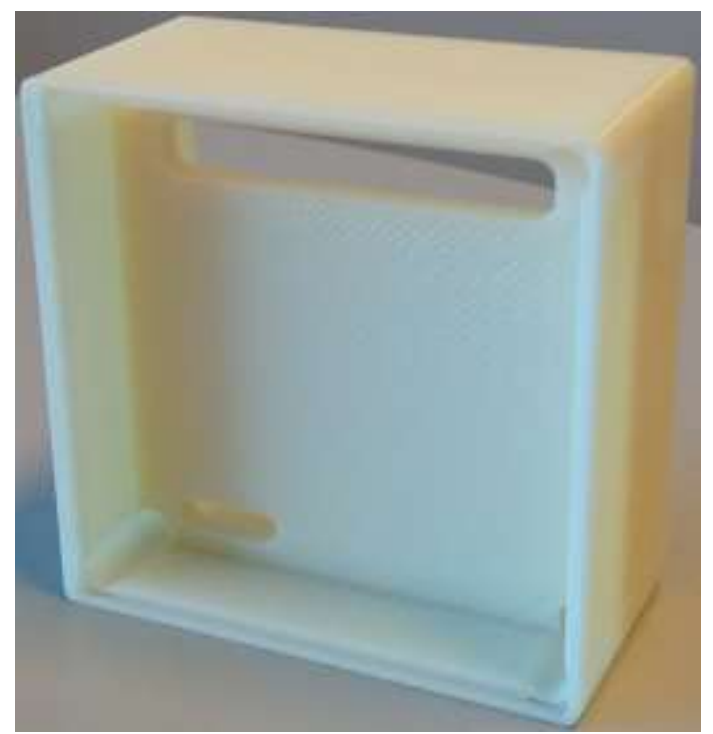

Figure 2: 3D printed version of $\mathrm{H}$-Section

The case was used in the mechanical engineering design course (ME 380 - Engineering Design Workshop) in fall 2013 to instruct 87 students. The course consisted of both lectures and other case study analyses. During the lectures, the topics in the competency material were discussed. That formed the basis for the discussion of the case studies by student teams during case delivery.

The course (ME380) is a third year design project core course. The Dalsa case was used to emphasize design for manufacturing concepts and provide a real-world application of manufacturing process analysis and cost estimation during product design. Students were provided with a sample 3D printed version of the mechanical part, Figure 2. The Dalsa Harmony Camera Design case study was assigned during the last week of the term after covering the needed theoretical concepts in the course.

The case and individual assignments were provided to the students one week in advance of the implementation date, after an introductory lecture on DFMA. During the individual assignment phase, questions were designed mainly to demonstrate students' ability to use appropriate knowledge and skills to: identify, formulate, analyze and develop substantiated solutions to a real-world problem. The students worked individually and in teams to analyze the problem and come up with their own 
recommendations about the manufacturing process and estimated cost. Often, individual study and preparation are followed by small group discussions, either in or outside of class. In this implementation, the class instructor (Nespoli) presented the case.

The class presentation focused on addressing most of the questions given to the students as part of their individual preparation assignments. Students were assigned to a team of 4 people and asked to discuss their individual answers in these teams in class, spending roughly 10 minutes on each question. The small group discussions facilitated the development of the students' ability to verbally communicate complex engineering concepts to group members. As part of the small group discussions, a number of different manufacturing methods for the H-section were suggested and discussed in class.

\section{STUDENT REACTION}

At the end of the case study in ME 380, students were asked to complete a feedback survey; 81 of 87 students responded. They were asked to respond to 5 questions on a Likert scale, from 1 (strongly disagree) to 5 (strongly agree). Figure 3 shows the student response to question 1: This case study was an engaging application of these specific course topics. The majority of students either agreed or strongly agreed with this statement. Engagement is a strong prerequisite for learning, and case studies provide a real-world application of course topics which students respond to. When asked to expand on their responses, students most often cited the strong fit between the case study and the course material. The numerical average of the student responses for this question was 4.3.

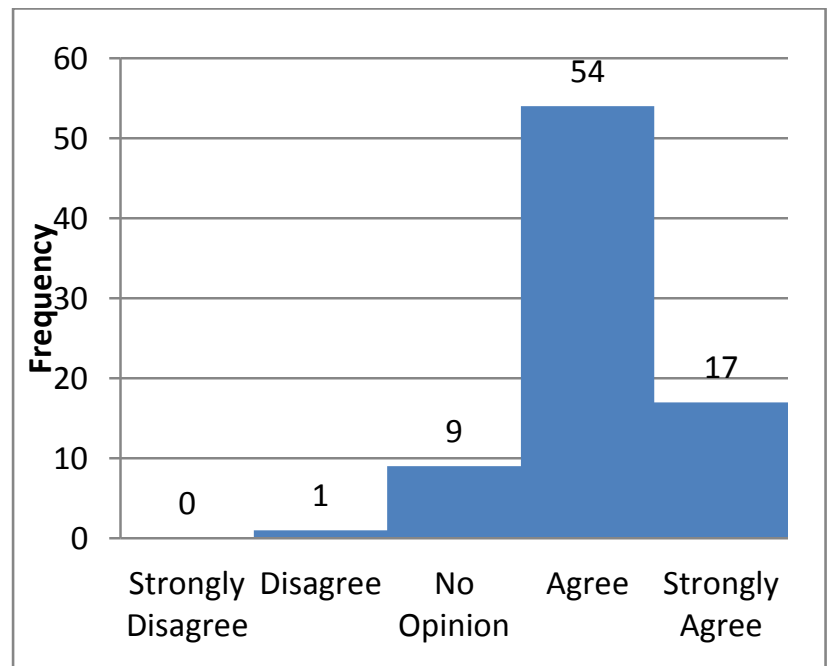

Figure 3: Student response to question 1: This case study was an engaging application of these course topics.
Question 2 further explored the connection between the case and the course material: This case study improved my appreciation for the relevance of these specific course topics. Similar results were obtained, with a numerical average of 4.2. Students highlighted the strong connection between the theoretical concepts discussed in class and the real-world practical example in the case.

Question 3 addressed student perception of their understanding of the material: This case study helped me understand these specific course topics. The numerical average of these responses was a little lower, 3.7. While students appreciated the application of course material, and one student commented on the increased perspective the case provided, a closer connection was desired. Two interesting responses were that a "high level [treatment] does not help understand low level concepts" and that while this was related to course content, "it feels unrelated since we don't have design questions outside of this." This hints that more time spent on the case and/or a design approach may help.

Question 4 addressed the perceived value of small group discussions: Small group discussions of the case helped me understand the specific course topics. Figure 4 shows the distribution of responses for this question. The numerical average of these responses was 3.6. Positive responses included the fact that the discussions "created an interactive environment with classmates" and "helped to clear the doubt and solved problems that couldn't be solved sitting alone." The primary reason for neutral or negative responses was the lack of time for these discussions.

This is an important point. To get the most out of this learning approach, more time is required for discussion. In business school applications, students are more familiar with the case approach and are trained to have these small group discussions before class, so that class time can be devoted to full class discussions. This is not feasible for the present situation, where only one or two cases are used in a class. This means that more class time should be set aside for small group discussions, which would require removal of some course content. A trade-off is necessary between content and case learning objectives. 


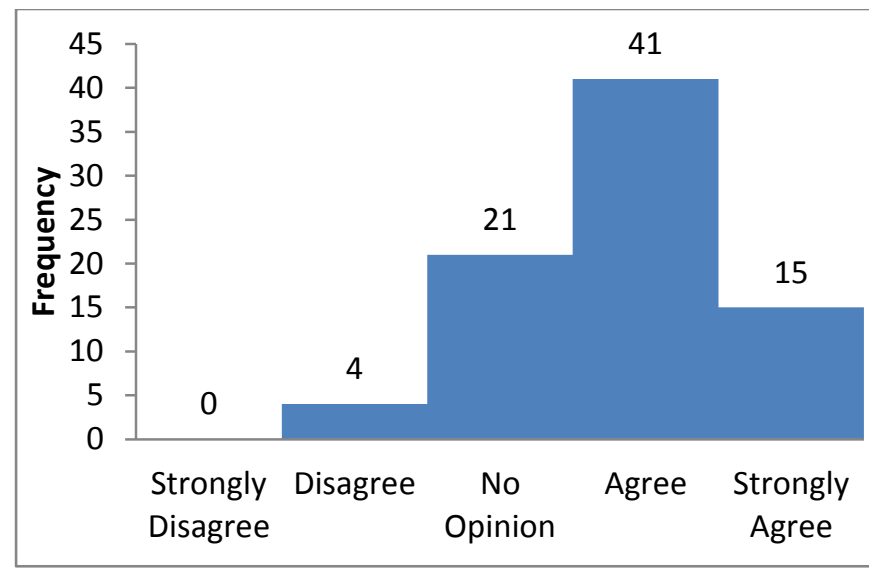

Figure 4: Student response to question 4: Small group discussions of the case helped me understand the specific course topics.

Students were also asked to provide general feedback for improvement of the case study and case activities. About half of the respondents suggested different time allocations. The amount of time available to present the case and the logistics should be cut back, including the presentation of background material, and the case should be introduced earlier in the term to allow for more guidance on what was expected. This would make the discussions more efficient. Some requested a more challenging problem, while others suggested a more openended topic, with less detail provided, might provide more scope for discussion.

\section{CONCLUSIONS}

Case studies offer a rich context to engage students in the development of engineering knowledge, skills and attitudes. We used WCDE case in the mechanical engineering design course (ME 380 - Engineering Design Workshop) in fall 2013. The results of the first implementation of this case study are encouraging. The students appreciated the added learning provided by this approximation to practice to lectures and examples provided by the instructor.

The 3D printed artifact was an important focus of the group discussions as confirmed by observations by the instructor. One of the 13 groups working on the case study arrived at the design solution adopted by Dalsa.

A possible extension of the case study would be to have groups estimate the manufacturing cost of the new process and materials.

\section{References}

[1]. Pahl G, Beitz W, Feldhusen J and Grote KH, Engineering Design - A Systematic Approach, 3rd edition, Springer, 2007

[2]. Ullman DG, The Mechanical Design Process, 4th Edition, McGraw-Hill, 2010

[3]. Harvard Business School, "The Case Method," 2007.

[4]. Paul G., Beitz W., Feldhusen J. and Grote K.H., "Engineering Design - A Systematic Approach", 3rd Edition, Springer, 2007, Available on-line: http://link.springer.com/book/10.1007/978-1-84628-3192/page/1

[5]. O. Molloy, E.A. Warman and S. Tilley "Design for Manufacturing and Assembly: Concepts, architectures and implementation" March 311998

[6]. Richard A Wysk, and Hsu-Pin Wang "Computer-Aided Manufacturing," Second Edition, Tien-Chien chang,. Pages 596 to 598. Prentice Hall 1998

[7]. Andrew Bogdanis and David Effa, "Dalsa Harmony Camera Design", WCDE 00221-TN, Waterloo Cases in Design Engineering (WCDE), University of Waterloo, 2013. 
APPENDIX A: Dalsa Camera Mechanical Part Fabrication Process Analysis Case Synopsis [7]

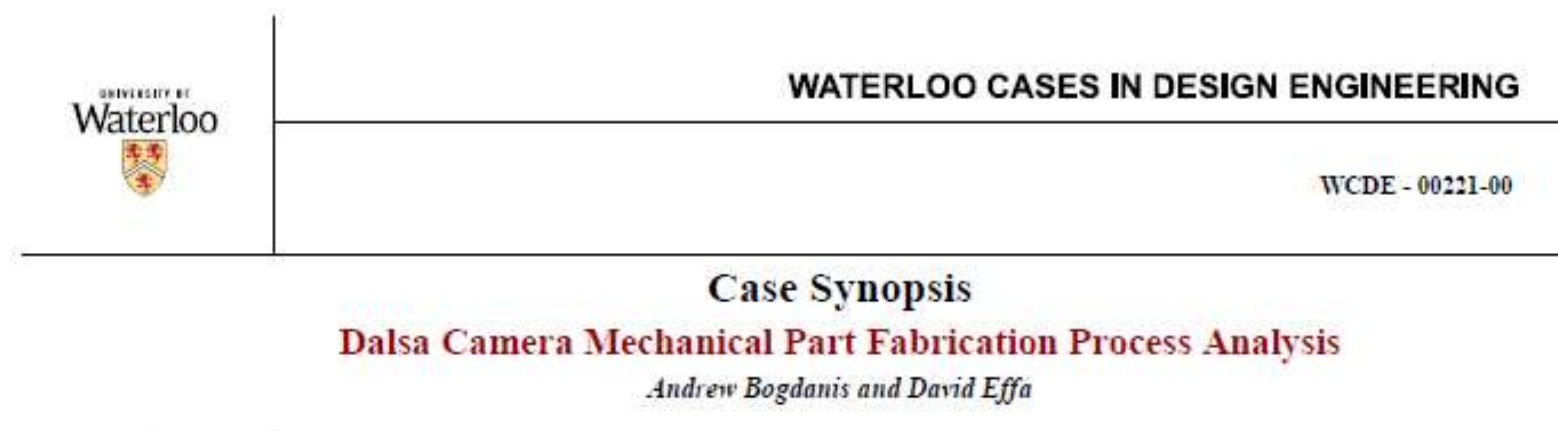

Subject Area: Manufacturing Processes, Heat Transfer, Thermal Design

Source: Teledyne Dalsa

\section{Description}

Teledyne Dalsa, headquartered in Canada, is a world leading high performance digital imaging and semiconductor business, providing engineering machine vision for a broad range of applications such as medical, industrial, aerospace, and defense systems. The Teledyne Dalsa Engineening Division provides complete engineering solutions for all camera and semiconductor projects. The division's projects are diversified in both application and industry, and can be either custom or standard depending on the customer's requirements. Recently, the engineering division planned to develop a new "Hanmony" camera for industrial image inspection applications. Harmony design is based on Dalsa's existing camera and specifically targets reduced production costs with comparable performance, scalable component architecture and lower BOM (bill of materials) cost. The project design focuses on the camera assembly, specifically reducing fabrication costs of the Hanmony camera body components (H-Section, shown in Figure 1), while maintaining adequate heat transfer performance. The H-section is the structural housing to which key electronic components are attached, and which transfers heat away from these components via an internal heat sink bridge.
Case Rerision Date: March 21, 2013

Length: 9 pages (Case Study)

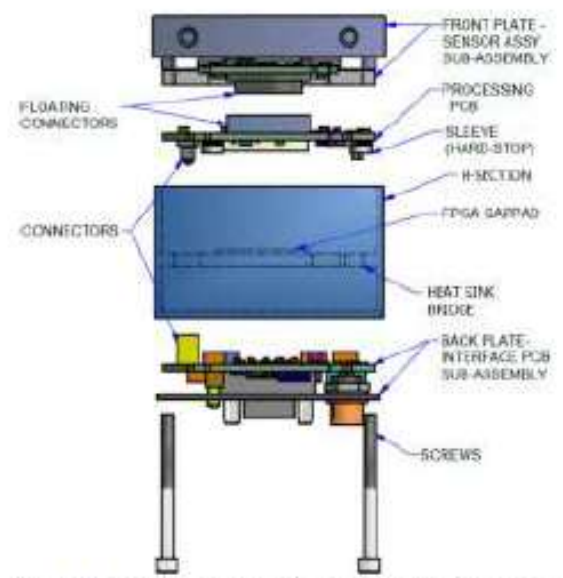

Figure 1:-Exploded view of the proposed Harmony camera

Andrew Bogdanis, a $4^{\text {th }}$ year mechanical engineening student from the University of Waterloo, was asked to assess the Hammony camera $\mathrm{H}$ Section parts fabrication process and perform a heat sink thermal analysis to verify conformance with requirements.

Learning Objectives:

This case study is intended for use in Mechanical Engineering and System Design courses covering topics such as thermodynamics and heat transfer. It can potentially be used in ME 322 - Mechanical Design 1, SYDE 361- Engineering Design and ME 353 Heat Transfer 1. The case study can also be used for other courses related to heat transfer mechanisms, including the formulation and solution of steady heat conduction problems.

Key Words: Punching Fixture; forming, machining; joining, Extrusion and Punching Method

CEAB Attribute: Design; Economics and Project Management; Problem Analysis; Use of Engineering Tools

Modules:

Module 01 - Case Study

Module 02 - Design Recuirements and Concept (Restricted to

educators only)

Module 03 - Thermal Design (Restricted to educators only)

Waterloo

WENGINEERING
Module 04 - Cost Saving Technology (Restricted to educators only)

Module TN - Teaching Note (Restricted to educators only) 